\title{
Epilepsy with hallucinatory phenomena in pediatric age: A case report
}

\author{
Marlene Rodrigues, Sofia Martins, Ana Antunes, Teresa Pontes, \\ Henedina Antunes, Ricardo Maré
}

\begin{abstract}
Introduction: Hallucinations in children may be part of normal development and not necessarily a symptom of mental illness, but they are subject of great concern to parents and clinicians. They may be a manifestation of a wide spectrum of disorders, such as intoxication, acute febrile illness, endocrinological, neurological or psychiatric disorder. Case Report: Clinical report of a previously healthy nine-year-old boy presented with visual and auditory hallucinations over the last month. During hospitalization it was performed electroencephalogram, audio record of hallucinatory description and child's illustration of the goblins seen in hallucinations. The etiological investigation excludes other causes, and changes in the electroencephalogram confirmed the diagnosis of epilepsy. The child presented with clinical improvement after treatment with sodium
\end{abstract}

Marlene Rodrigues ${ }^{1}$, Sofia Martins ${ }^{2}$, Ana Antunes ${ }^{2}$, Teresa Pontes $^{2}$, Henedina Antunes ${ }^{3}$, Ricardo Maré ${ }^{4}$

Affiliations: ${ }^{1}$ Pediatric Resident, Pediatrics Department, Hospital de Braga, Braga, Portugal; '2Pediatric Consultant, Pediatrics Department, Hospital de Braga, Braga, Portugal; ${ }^{3}$ Pediatric Gastroenterology Senior Consultant and Professor of Pediatrics, Pediatrics Gastroenterology, Hepatology and Nutrition Unit, Hospital de Braga and Life and Health Sciences Research Institute (ICVS), Health Sciences School of University of Minho, Associated Laboratory ICVS/3B's, Braga/Guimarães, Portugal; ${ }^{4}$ Neurology Consultant, Neurology Department, Hospital de Braga, Portugal.

Corresponding Author: Marlene Rodrigues, Hospital de Braga, Sete Fontes - São Victor, 4710-243 Braga, Portugal; Email: rodrigues.f.marlene@gmail.com

Received: 07 August 2016

Accepted: 19 November 2016

Published: 19 December 2016 valproate, without recurrence of symptoms. Conclusion: This clinical report intended to emphasize the importance of considering epilepsy as a differential diagnosis in children and adolescents with psychic phenomenology, to make a timely diagnosis and treatment.

Keywords: Children, Epilepsy, Hallucinations, Pediatrics

\section{How to cite this article}

Rodrigues M, Martins S, Antunes A, Pontes T, Antunes H, Maré R. Epilepsy with hallucinatory phenomena in pediatric age: A case report. Case Rep Int 2016;5:44-47.

Article ID: 100030CRINTMR2016

$* * * * * * * * *$

doi:10.5348/crint-2016-30-CR-11

\section{INTRODUCTION}

In adults, hallucinations are usually linked to serious psychopathology but in children they are less frequents and not necessarily a symptom of mental disease [1].

A hallucination is a sensory perception in the absence of an adequate external stimulus. They can be elementary as hearing a sound or see a point of light, usually originating in the occipital lobe, or complex, like listening sounds, someone speaking or watching structured pictures/scenes that can occur on seizures originated in occipital, temporal (mesial or mesocortical) or parietal lobes and limbic area [2, 3]. In some cases, the origin of hallucinations is not cortical and may arise from brainstem and amygdala dysfunction. They must be distinguished from similar phenomena such as illusions 
(altered perception of a real external stimulus), elaborated fantasies and imaginary people $[2,4,5]$.

Hallucinations in childhood may be triggered in the course of some acute diseases, sometimes also associated with altered state of consciousness, such as febrile illness and intoxications. They can also be a manifestation of neurological diseases, such as migraine or epilepsy, and psychiatric as schizophrenia, depression and anxiety [68].

Hallucinations caused by convulsive disorders are rare and can be somatosensory, visual, auditory, olfactory or gustatory. The diagnosis involves a detailed clinical history and physical examination. Electroencephalogram (EEG) is mandatory, as well as brain magnetic resonance imaging. Hallucinations approach involves the treatment of underlying primary disease $[1,7-9]$.

\section{CASE REPORT}

We report a previously healthy nine-year-old boy, with a family history of epilepsy in a paternal aunt, admitted to emergency service of residence area with persistent daily auditory hallucinations, sometimes also visual, occurring in bursts over the last month. He referred that had seen colored monsters and goblins that attacked, screamed and told him to go away; Sometimes he tried to hide himself under the bedclothes so he could not see them and other times he was found closed in the bathroom crying. It was not identified any factor that make seizures worse or better and no postictal symptoms were described.

The patient had one isolated determination of fever and diarrhea with no other associated symptoms, at admission day. Analytical study including blood count, ionic and acid-base balance, renal function, transaminases, C-reactive protein and blood cultures did not reveal alterations, as well as urinalysis with negative urine culture.

The patient was transferred to our hospital institution for neurology evaluation. On admission, he was thermodynamically stable with normal physical and neurological examination. Lumbar puncture was performed (cells 2, erythrocytes o, proteins $0.18 \mathrm{~g} / \mathrm{L}$ ) with negative virological (enterovirus, herpes I and II, varicella zoster, cytomegalovirus, Epstein-Barr virus) and microbiological of cerebrospinal fluid. Brain magnetic resonance imaging scan was normal.

During hospitalization, after informed consent of legal representative, audio record of hallucinatory description and child's illustration of the goblins seen in hallucinations were made (Figure 1). Video-EEG revealed left and right side epileptiform activity on temporalparietal-occipital areas (not synchronous). The child was cooperative during the examination. No specific changes were evidenced by hyperpnea and intermittent light stimulation. Neither epileptic seizures nor behavioral changes were observed.
The diagnosis of simple partial epilepsy with psychic symptoms (complex visual and/or hearing hallucinations and intense feeling of fear) was established. $\mathrm{He}$ had favorable outcome with complete remission of hallucinatory episodes after sodium valproate therapy with dosage of $15 \mathrm{mg} / \mathrm{kg}$ per day.

The child is being followed-up on consultation and maintains therapy with sodium valproate $20 \mathrm{mg} / \mathrm{kg}$ per day. No further episodes of hallucinations were registered. The EEG in the last follow-up, nine months after the diagnosis, showed scarce epileptiform activity on the right centro-parietal area (Figure 2).

\section{DISCUSSION}

Hallucinations in children are subject of great concern and anxiety to parents and clinicians. In adults, they are often synonymous of psychotic illness, but in children

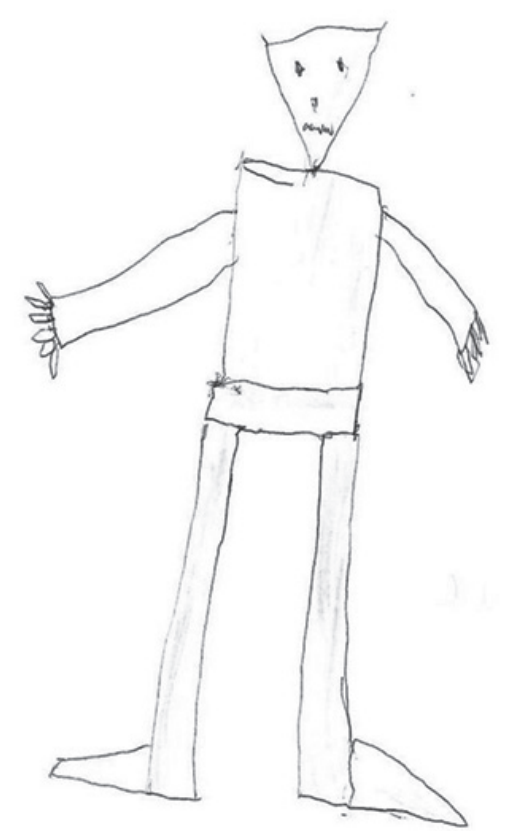

Figure 1: Child's illustration representing goblins seen in hallucinations.

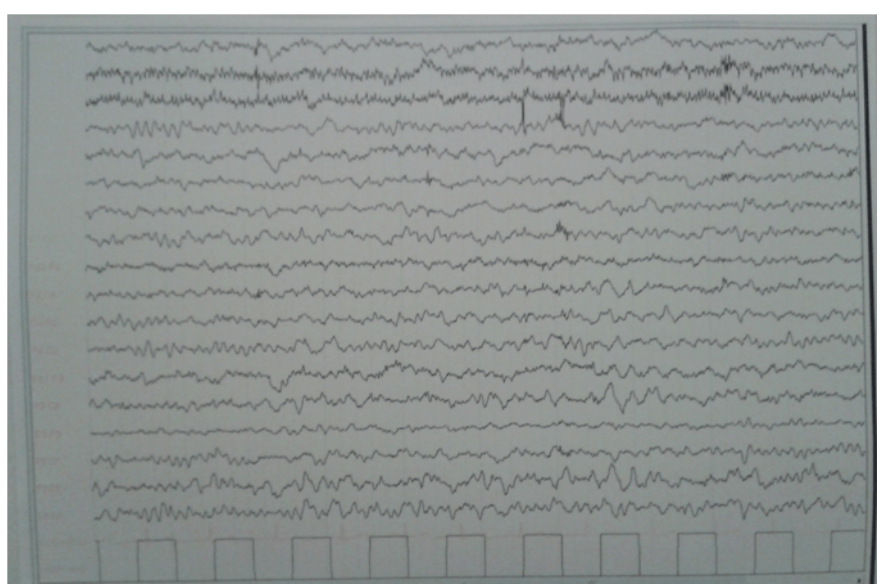

Figure 2: Electroencephalogram of patient. 
can be part of normal development or an acute disease manifestation [1].

In epileptic patients, neuronal discharges at occipital, temporal or parietal lobes and limbic area may result in ictal psychotic phenomena. Psychic phenomenology of epileptic nature must be considered in differential diagnosis of a number of other entities. In a study of 62 children with nonpsychotic source hallucinations, 34\% had depression, 22\% hyperactivity disorder and attention deficit, 21\% behavioral disturbances and 23\% other diagnosis [1].

The case we report is an example in which hallucinations are the expression of an underlying disease, in this case epilepsy. Auditory hallucinations indicate, most likely, temporal dysfunction while the complex visual hallucinations, evoking visual memories, are associated with activation of parietal-temporal visual association cortex [9-11]. The fear associated with these phenomena can be related to the hallucination content and/or activation of limbic areas. Seizures associated with hallucinations and fear are often difficult to distinguish from psychiatric disorders, and psychological symptoms are sometimes the "top of the iceberg symptoms," pointing to some less obvious cause. They can mimic a number of other pathologies, delaying the correct diagnosis. Therefore a proper investigation of such symptoms is mandatory.

In our case, video-EEG was performed and proved to be essential to confirm the diagnosis, detecting ictal activity, although without observed seizures or behavior disturbs during the examination test. However, it is important to note that EEG is not always able to detect ictal activity in this case, so the video documentation of hallucinations can help to uncover important signs for the differential diagnosis [8].

\section{CONCLUSION}

Hallucinations in children and adolescents requires a rigorous clinical examination, diagnostic tests and detailed psychological evaluation, to rule out medical causes and identify the psychopathological, psychosocial and cultural factors that may be associated.

\section{Author Contributions}

Marlene Rodrigues - Substantial contributions to conception and design, Acquisition of data, Analysis and interpretation of data, Drafting the article, Revising it critically for important intellectual content, Final approval of the version published

Sofia Martins - Analysis and interpretation of data, Acquisition of data, Revising it critically for important intellectual content, Final approval of the version to be published
Ana Antunes - Analysis and interpretation of data, Revising it critically for important intellectual content, Final approval of the version to be published

Teresa Pontes - Analysis and interpretation of data, Revising it critically for important intellectual content, Final approval of the version to be published

Henedina Antunes - Analysis and interpretation of data, Revising it critically for important intellectual content, Final approval of the version to be published

Ricardo Maré - Substantial contributions to conception and design, Analysis and interpretation of data, Acquisition of data, Revising it critically for important intellectual content, Final approval of the version to be published

\section{Guarantor}

The corresponding author is the guarantor of submission.

\section{Conflict of Interest}

Authors declare no conflict of interest.

\section{Copyright}

(C) 2016 Marlene Rodrigues et al. This article is distributed under the terms of Creative Commons Attribution License which permits unrestricted use, distribution and reproduction in any medium provided the original author(s) and original publisher are properly credited. Please see the copyright policy on the journal website for more information.

\section{REFERENCES}

1. Sidhu K, Dickey T. Hallucinations in children: Diagnostic strategies. Current Psychiatry 2010;9(10):53-60.

2. Kasper BS, Kasper EM, Pauli E, Stefan H. Phenomenology of hallucinations, illusions, and delusions as part of seizure semiology. Epilepsy Behav 2010 May;18(1-2):13-23.

3. González-Cuevas M, Toledo M, Santamarina E, et al. Visual epileptic seizures. Signs and symptoms, and clinical implications. [Article in Spanish]. Rev Neurol 2015 Mar 16;60(6):257-62.

4. Elliott B, Joyce E, Shorvon S. Delusions, illusions and hallucinations in epilepsy: 1. Elementary phenomena. Epilepsy Res 2009 Aug;85(2-3):162-71.

5. Elliott B, Joyce E, Shorvon S. Delusions, illusions and hallucinations in epilepsy: 2. Complex phenomena and psychosis. Epilepsy Res 2009 Aug;85(2-3):17286.

6. Garralda ME. Fifteen minute consultation on children 'hearing voices': When to worry and when to refer. Arch Dis Child Educ Pract Ed 2015 Oct;100(5):233-7.

7. Perena H, Attygale U, Jeewandara C, Jayawardena V. Non-psychotic auditory hallucinations in children and adolescents. Sri Lanka Journal of Psychiatry 2011;2(1):9-12.

8. Kechid G, Auvin S, Jardri R, Vallée L, Delion P, Goëb JL. Hearing hallucinations in a 12-year-old child: 
Psychotic disorders or temporal epilepsy? Prim Care Companion J Clin Psychiatry 2008;10(4):328-9.

9. Weber P, Ruof H, Jourdan S. Differential diagnosis of visual hallucinations. [Article in German]. Klin Padiatr 2005 Jan-Feb;217(1):25-30.

10. Florindo I, Bisulli F, Pittau F, et al. Lateralizing value of the auditory aura in partial seizures. Epilepsia 2006;47 Suppl 5:68-72.
11. La Vega-Talbot M, Duchowny $M$, Jayakar $P$. Orbitofrontal seizures presenting with ictal visual hallucinations and interictal psychosis. Pediatr Neurol 2006 Jul;35(1):78-81.
Access full text article on other devices

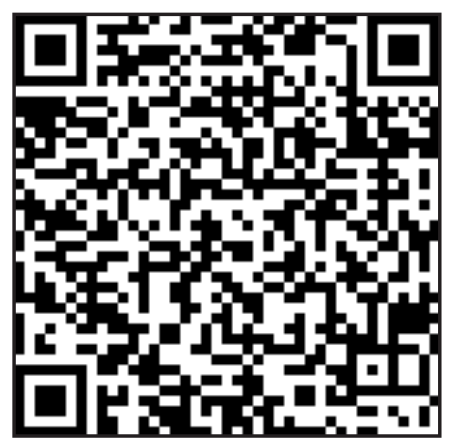

Access PDF of article on other devices

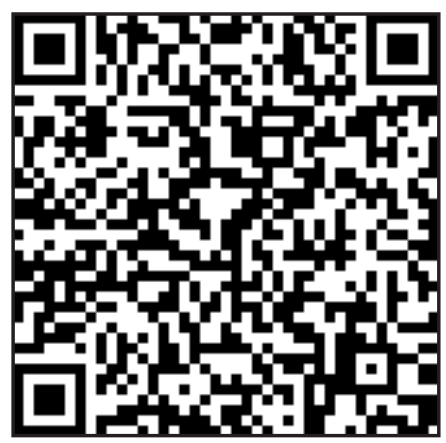

